\title{
Redo hypospadias repair: experience at a tertiary care children's hospital
}

\author{
Ahmed Al-Sayyad, MD; John G. Pike, MD; Michael P. Leonard, MD
}

\begin{abstract}
Objective: Treatment of patients with failed hypospadias repairs can be challenging. Our study aimed to determine the best type of redo repair depending on the location and size of the urethral meatus, the status of the urethral plate and genital skin, the severity of residual chordee and the amount of scar tissue.
\end{abstract}

Methods: The Institutional Review Board approved our retrospective chart review of patients who had a redo hypospadias repair at our institution over the past 6 years. We recorded the type and number of previous repair(s), the type and number of redo procedure(s), as well as the complications and functional outcomes.

Results: There were 28 patients, aged 1-12 (mean 3.8) years, with failed hypospadias repairs. The initial severity of the hypospadias were as follows: perineal (1), penoscrotal (9), proximal shaft (1), mid-shaft (9), distal shaft (4), coronal (3) and mega-meatus (1). Of all the patients, 24 had 1 repair, 3 had 2 repairs and 1 had 3 repairs. The initial repairs comprised 11 tubularized island flaps (TIFs), 8 Snodgrass tubularized incised plate (TIP) techniques, 5 Mathieu repairs, 1 Meatal Advancement and GlanuloPlasty Incorporated (MAGPI) technique, 1 Pyramid, 1 Arap technique and 1 Thiersch-Duplay repair. Twenty-one of 28 patients had 1 redo operation, 5 had 2 redo operations, 1 had 3 redo operations and 1 had 4 redo operations, for a total of 38 redo operations. Of these, 26 were TIP techniques $(68.4 \%), 3$ were Mathieu $(7.9 \%)$, 3 were TIF repairs $(7.9 \%), 2$ were onlay island flaps $(5.3 \%)$ and 4 were buccal mucosal grafts (10.5\%). Follow-up was $1-5$ years (mean $3.5 \mathrm{yr}$ ). The final locations of urethral meatus included glans (18), corona (6), mid-shaft (3) and penoscrotal (1). Complications after redo surgery comprised 4 urethrocutaneous fistulae, 2 meatal stenoses, 1 urethral stricture and 3 dehiscences. Sixteen patients were followed with yearly uroflow with a Q-mean (mean uroflow) range of 3-14 mL/s (mean $8.1 \mathrm{~mL} / \mathrm{s})$.

Conclusion: The majority of hypospadias failures can be salvaged with one operation. The TIP repair is our procedure of choice in most cases. In the setting of a poor urethral plate, TIF or buccal mucosa may be necessary. Complications are not infrequent in redo procedures.

\section{CUAJ 2007;1(1):47-51}

n current practice, hypospadias repairs are performed as outpatient procedures with high success rates. Despite the use of optical magnification, fine suture material and modern techniques, we still encounter the occasional failure that requires reoperation. Secondary operations can be challenging because of scarring and the paucity of penile skin. We review our experience with redo hypospadias operations at the Children's Hospital of Eastern Ontario.

\section{Methods}

The Institutional Review Board approved our retrospective chart review of patients who underwent redo hypospadias repair at the Children's Hospital of Eastern Ontario over the past 6 years. We looked at the number and type of initial and redo repairs they had. A minimum of at least 6 months was allowed to elapse between operations. To formulate an idea of the type of redo repair we would use, the clinical examination focused on the location and size of the urethral meatus, the status of the urethral plate and genital skin, the severity of residual chordee and the amount of scarring. We recorded complications and functional outcomes of redo procedures by performing uroflowmetry.

\section{Results}

We reviewed 28 patients who ranged in age from 1 to 12 years (mean $3.8 \mathrm{yr}$ ). The reason for redo surgery was complete dehiscence in 27 patients and urethral stricture in 2 patients ( 1 patient had both complete dehiscence and a urethral stricture). The initial severity of the hypospadias was perineal in 1 patient $(3.6 \%)$, penoscrotal in 9 patients $(32.1 \%)$, proximal shaft in 1 patient $(3.6 \%)$, mid shaft in 9 patients $(32.1 \%)$, distal shaft in $4(14.3 \%)$, coronal in $3(10.7 \%)$ and megameatus in 1 patient (3.6\%) (Fig. 1). There was 1 previous attempt at repair in $85.7 \%$ of patients. There were 2 and 3 previous attempts at repair in $10.7 \%$ and $3.6 \%$ of patients, respectively (Table 1). The type of initial repair comprised 39.9\% tubularized island flap (TIF) 
repair, 28.5\% tubularized incised plate (TIP) repair, $17.8 \%$ Mathieu repair, 3.6\% Meatal Advancement and GlanuloPlasty Incorporated (MAGPI) technique, 3.6\% Pyramid repair, $3.6 \%$ Arap technique and $3.6 \%$ Thiersch-Duplay repair (Fig. 2). Of patients, $21(75 \%)$ underwent 1 redo operation, $5(17.8 \%)$ underwent 2 redo operations, $1(3.6 \%)$ had 3 redo operations and 1 patient $(3.6 \%)$ had 4 redo operations. A total of 38 redo operations were performed in our 28 patients as follows: 26 $(68.4 \%)$ TIP repairs, $3(7.9 \%)$ Mathieu repairs, $3(7.9 \%)$ TIF repairs, $2(5.3 \%)$ onlay island flap (OIF) repairs and 4 $(10.5 \%)$ buccal mucosal grafts (Fig. 3$)$. Follow-up ranged from 1 to 5 years with a mean of 3.5 years. Final location of the meatus was glans in 18 cases, corona in
6 cases, mid shaft in 3 and penoscrotal in 1 case.

Urethrocutaneous fistulae occurred in 4 patients ( 3 TIP and 1 TIF) and were treated successfully with surgical closure. Meatal stenosis in occurred in 2 patients after TIP and both were treated with simple meatotomy. Urethral stricture was seen in 1 patient after buccal mucosa graft and required a cut back urethroplasty with plans for future reconstruction. Complete dehiscence occurred in 3 patients (2 TIP and 1 buccal graft) who are awaiting further surgery (Table 2). Sixteen of our patients were followed with yearly uroflow, with a Q-mean range of 3-14 mL/s (mean $8.1 \mathrm{~mL} / \mathrm{s}$ ). Uroflow was within normal range (standard deviation [SD] 2) in $100 \%$ of patients, with only

\begin{tabular}{|c|c|c|}
\hline Procedure & Dehiscence & Stricture \\
\hline TIF & 12 & 1 \\
\hline TIP & 7 & 1 \\
\hline MAGPI & 1 & 0 \\
\hline Mathieu repair & 6 & 0 \\
\hline Pyramid & 1 & 0 \\
\hline Arap technique & 1 & 0 \\
\hline $\begin{array}{l}\text { Thiersch-Duplay } \\
\text { repair }\end{array}$ & 3 & 0 \\
\hline \multicolumn{3}{|c|}{$\begin{array}{l}\text { TIF = tubularized island flap; TIP = tubularized incised plate; } \\
\text { MAGPI = meatal advancement and glanuloplasty incorporated } \\
\text { technique. }\end{array}$} \\
\hline
\end{tabular}

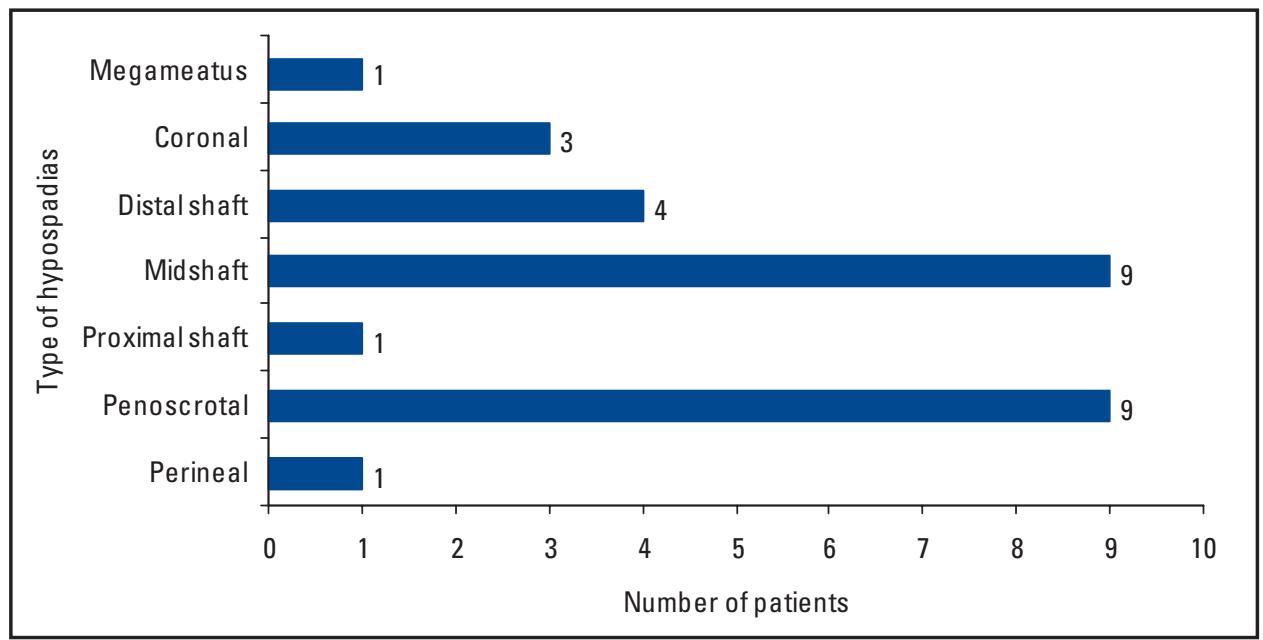

Fig. 1. Severity of initial hypospadias. 
2 patients averaging below the fiftieth percentile. Postvoid residual volume greater than $10 \%$ of voided volume was seen in 2 patients.

\section{Discussion}

The treatment of failed hypospadias can be challenging because of significant scar-

Table 2: Type of redo procedures and their respective complications

\begin{tabular}{lcccc} 
Procedure & UC fistula & Stricture & $\begin{array}{c}\text { Meatal } \\
\text { stenosis }\end{array}$ & Dehiscence \\
\hline TIP & 3 & 0 & 2 & 2 \\
TIF & 1 & 0 & 0 & 0 \\
Buccal mucosa & 0 & 1 & 0 & 1 \\
UC = urethrocutaneous; TIP $=$ tubularized incised plate; TIF = tubularized island flap. & \\
\hline
\end{tabular}

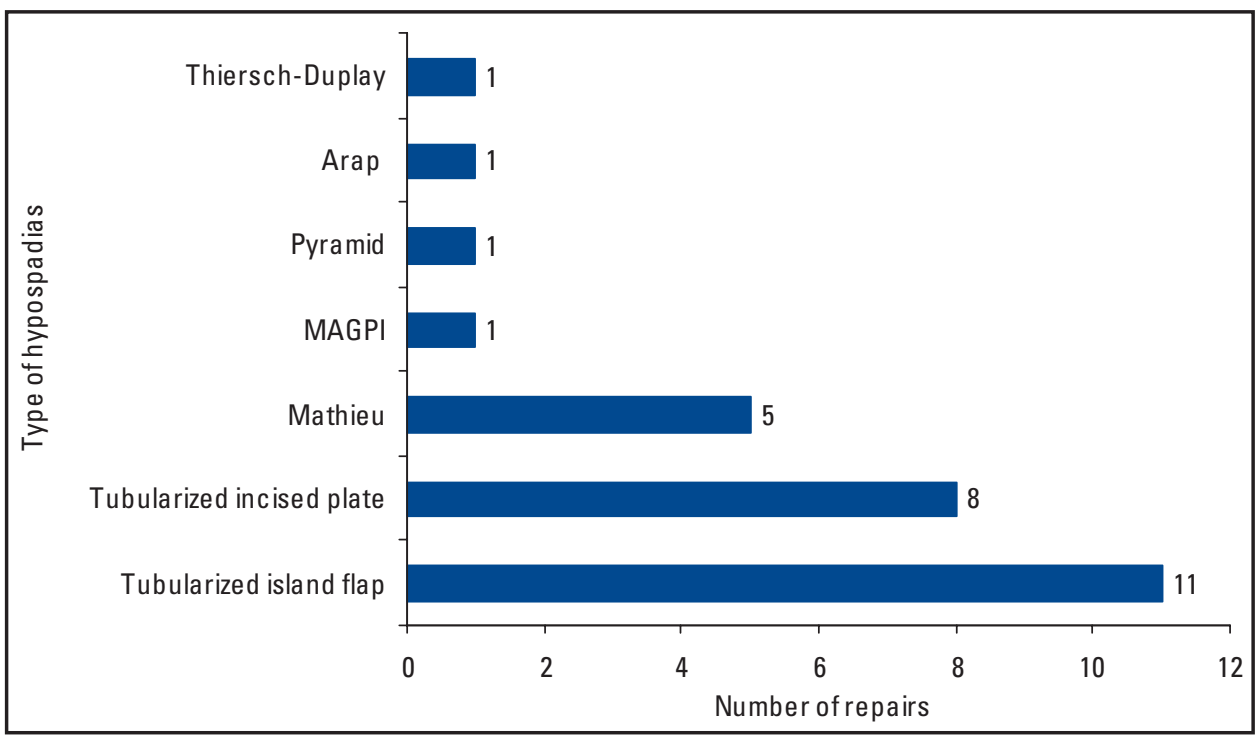

Fig. 2. Prior hypospadias repairs. MAGPI = Meatal Advancement and GlanuloPlasty Incorporated technique.

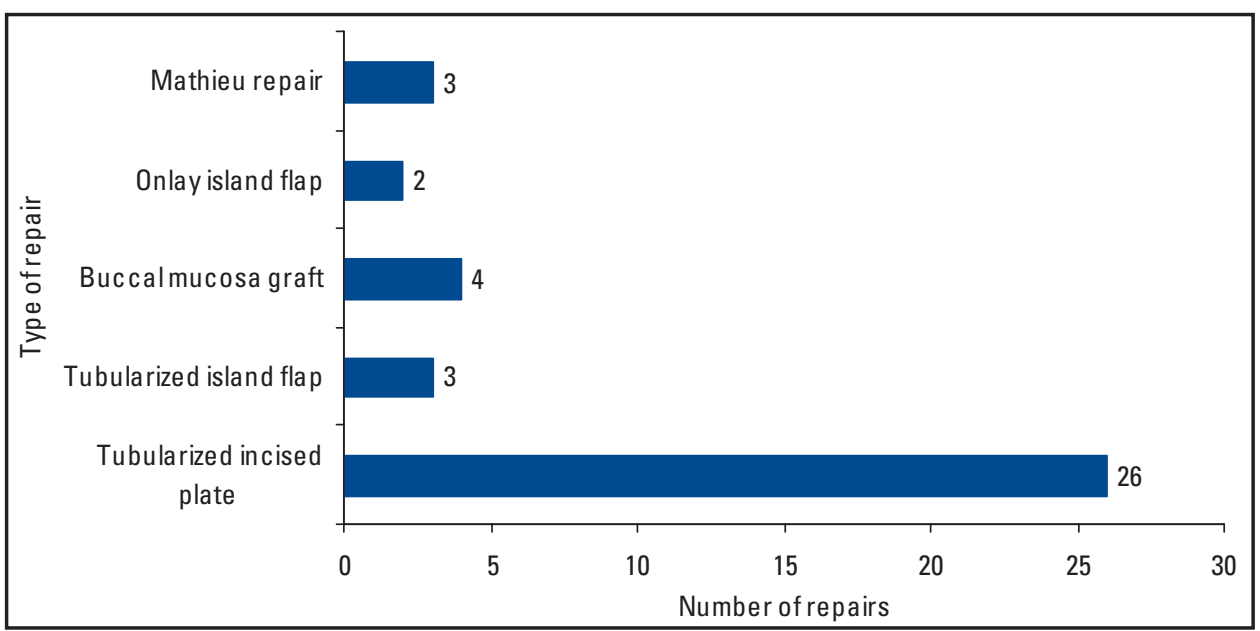

Fig. 3. Types of redo procedures. 
ring and paucity of genital skin; thus, leaving the surgeon with less than ideal material with which to reconstruct the patient. Different modes for salvage techniques have been reported, including TIP urethroplasty, the Mathieu repair, onlay and tubularized island flaps, and free grafts. Snodgrass and Lorenzo reported their initial experience of using TIP urethroplasty in redo hypospadias repairs. They had 15 patients with a mean follow-up of 5 months. ${ }^{1}$ They achieved a cosmetically normal meatus in 13 of 15 patients and complications comprised 2 fistulae and 1 glans dehiscence. Borer and colleagues ${ }^{2}$ also used TIP in 25 redo cases with satisfactory results. Complications included 5 fistulae and 1 meatal stenosis. ${ }^{2}$ Shanberg and colleagues also reported TIP urethroplasty in 13 redo cases with a mean follow-up of 22 months. Cosmetic results were excellent, with 2 complications: one patient with a glans dehiscence and a urethrocutaneous fistula, and a second patient who developed meatal stenosis. ${ }^{3}$ Simmons and colleagues reported their series of 53 patients for whom they used a Mathieu flip-flap in 17 repairs and an OIF in 36 repairs. They had a successful salvage in $83 \%$ of their patients using a single procedure. ${ }^{4}$ Jayanthi and colleagues, and Secrest and colleagues also showed good outcome using flap techniques. ${ }^{5,6}$ In our series, a meatus situated on the glans was achieved in $64 \%$ of patients. However, a meatus situated at the corona allows for normal penile function, enabling the patient to void standing. Thus, one could consider a meatus situated on the glans or at the corona a success, which would then have been achieved in $86 \%$ of our cases.

Initial examination of these patients usually guides our decision of which salvage technique could be used. However, the final decision is usually made intraoperatively after taking into consideration the amount and location of scarring, the availability and quality of penile skin, and the quality of the urethral plate. General principles of hypospadias repair should be followed, including, minimal use of cautery, avoidance of tension, use of well vascularized tissue, closure in as many layers as possible, the use of loupe magnification and identification, and the relief of any obstruction. If the urethral plate is present and wide enough, we elect for a TIP urethroplasty. Using this technique, we have had satisfactory results comparable with previously mentioned series..$^{1-3}$ If the urethral plate is not suitable for a TIP or if it is absent and there is enough penile skin, we perform an OIF or a TIF. If there is a grossly scarred urethral plate contributing to chordee, it should be excised and replaced with a buccal mucosa graft for a 2-stage repair as advocated by Bracka.? We performed 2 onestage buccal mucosa repairs and we had total failure using this technique. Although our conclusion is based on a small number of patients, we have stopped using 1-stage buccal mucosa grafting. The experience of the Children's Hospital of Philadelphia group, which reported complications in over one-half of their patients using 1-stage buccal mucosa repairs, echoes our concerns. ${ }^{8}$

Sixteen of our patients were followed with yearly uroflow. The remainder of the group was either lost to follow-up or was too young to be able to perform uroflowmetry (i.e., not yet toilet trained). Uroflow was within normal range (SD 2) in $100 \%$ of patients followed. Hammouda and colleagues also reported normal uroflow in redo hypospadias; they had normal flow in 7 of $9(77.8 \%)$ redo patients. ${ }^{9}$

\section{Conclusions}

Redo hypospadias repairs are challenging and occasionally humbling; urethroplasty should attempt to incorporate the urethral plate. If the urethral plate is not available and there is inadequate penile skin to fashion a TIF, then buccal mucosa is the free graft material of choice. The need for subsequent procedures to deal with complications is a reality. Eventually, 
excellent functional and cosmetic results can be attained if one persists in addressing this difficult problem.

From the Division of Pediatric Urology, Children's Hospital of Eastern Ontario, University of Ottawa, Ottawa, Ont.

This article has been peer reviewed.

Competing interests: None declared.

\section{References}

1. Snodgrass WT, Lorenzo A. Tubularized incised-plate urethroplasty for hypospadias reoperation. BJU Int 2002;89:98-100.

2. Borer JG, Bauer SB, Peters CA, et al. Tubularized incised plate urethroplasty: expanded use in primary and repeat surgery for hypospadias. J Urol 2001;165:581-5.

3. Shanberg AM, Sanderson K. Duel B. Reoperative Hypospadias repair using the Snodgrass incised plate urethroplasty. BJU Int 2001;87:544-7.
4. Simmons GR, Cain MP, Casale AJ, et al. Repair of hypospadias complications using the previously utilized urethral plate. Urology 1999; 54:724-6.

5. Jayanthi VR, McLori GA, Khoury AE, et al. Can previously relocated penile skin be successfully used for salvage hypospadias repair? J Urol 1994; 152:740-3.

6. Secrest $\mathrm{CL}$, Jordan $\mathrm{GH}$, Winslow BH, et al. Repair of the complications of hypospadias surgery. J Urol 1993;150:1415-8.

7. Bracka A. Hypospadias repair: the two-stage alternative. Br J Urol 1995; 76(Suppl.3):31-41.

8. Metro MJ, Wu HY, Snyder HM, et al. Buccal mucosal grafts: lessons learned from an 8-year experience. J Urol 2000;166:1459-61.

9. Hammouda H, El-Ghonemi A, Khoury A. Tubularized incised plated repair: functional outcome after intermediate follow up. J Urol 2003;169:331-3.

Correspondence: Dr. Michael Leonard, Division of Urology, Children's Hospital of Eastern Ontario, 401 Smyth Rd., Ottawa ON KIH 8LI; mleonard@cheo.on.ca 